\title{
Ensino das práticas integrativas e complementares em saúde na enfermagem
}

\author{
Teaching integrative and complementary practices in nursing \\ Enseñanza de prácticas integrativas y complementarias en salud en enfermería
}

Recebido: 04/01/2022 | Revisado: 11/01/2022 |Aceito: 15/01/2022 | Publicado: 17/01/2022

\author{
Thaiane Santana Santos \\ ORCID: https://orcid.org/0000-0003-2207-8055 \\ Universidade Federal de Sergipe, Brasil \\ E-mail: thaianesantana08@gmail.com \\ Laura Dayane Gois Bispo \\ ORCID: https://orcid.org/0000-0003-1628-520X \\ Universidade Federal de Sergipe, Brasil \\ E-mail: lauradayane2010@hotmail.com \\ Iellen Dantas Campos Verde Rodrigues \\ ORCID: https://orcid.org/0000-0001-8841-669X \\ Universidade Federal do Piauí, Brasil \\ E-mail: iellendantas@hotmail.com \\ Deborah Danielle Tertuliano Marinho e Albuquerque \\ ORCID: https://orcid.org/0000-0001-8251-5240 \\ Universidade Federal de Sergipe, Brasil \\ E-mail: deborahdani5@yahoo.com.br \\ Carla Kalline Alves Cartaxo Freitas \\ ORCID: https://orcid.org/0000-0001-7604-9132 \\ Universidade Federal de Sergipe, Brasil \\ E-mail: carlakalline@academico.ufs.br
}

\begin{abstract}
Resumo
Objetivo: Descrever a experiência do ensino das Práticas Integrativas e Complementares em Saúde (PICS) na graduação em enfermagem. Método: Trata-se de um relato de experiência sobre a disciplina das PICS, realizada em ambiente virtual, síncrono, no curso de enfermagem da Universidade Federal de Sergipe. Resultados: A disciplina proporcionou um conteúdo inovador, essencial para a compreensão do que realmente significa o cuidado integral, bem como o conhecimento de todas as PICS disponíveis no Sistema Único de Saúde e suas possibilidades nas ações em saúde e na assistência de enfermagem. Ademais, ela propôs estratégias efetivas para o autocuidado, de forma a melhorar a qualidade de vida dos acadêmicos. Conclusão: A experiência do processo de ensino-aprendizagem das PICS no curso de enfermagem foi exitosa, o que fomenta a adesão da mesma nas demais Instituições de Ensino Superior. Ensinar PICS é contribuir com a implementação dos princípios do SUS, impulsionando a integralidade do cuidado em saúde.
\end{abstract}

Palavras-chave: Terapias complementares; Ensino; Enfermagem; Sistema Único de Saúde.

\begin{abstract}
Objective: To disseminate the experience of teaching Integrative and Complementary Practices in Health (PICS) in undergraduate courses with the disease. Method: This is an experience report in the PICS discipline, carried out in a synchronous virtual environment, not in a nursing course at the Federal University of Sergipe. Results: The course provided innovative content, essential for understanding what comprehensive care really means, as well as knowledge of all the PICS available to them by Sistema Único de Saúde and their possibilities for health and nursing care. In addition, it makes effective self-care strategies in order to improve the quality of life of two students. Conclusion: The experience of the PICS teaching-learning process was not a successful nursing course, or one that encourages the continuation of teaching in Higher Education Institutions. To teach PICS is to contribute to the implementation of two SUS principles, promoting comprehensive health care.
\end{abstract}

Keywords: Complementary therapies; Teaching; Nursing; Health Unic System.

\section{Resumen}

Objetivo: Difundir la experiencia de la docencia de Prácticas Integrativas y Complementarias en Salud (PICS) en cursos de pregrado con la enfermedad. Método: Se trata de un relato de experiencia en la disciplina PICS, realizado en un entorno virtual sincrónico, no en un curso de enfermería de la Universidad Federal de Sergipe. Resultados: El curso brindó contenidos innovadores, imprescindibles para comprender lo que realmente significa la atención integral, asî como el conocimiento de todos los PICS a su disposición en lo Sistema Único de Saúde y sus posibilidades para la salud y la atención de enfermería. Además, realiza estrategias efectivas de autocuidado con el fin de mejorar la calidad de vida de dos alumnos. Conclusión: La experiencia del proceso de enseñanza-aprendizaje del PICS no fue un curso de 
enfermería exitoso, ni que incentive la continuación de la docencia en las Instituciones de Educación Superior. Enseñar PICS es contribuir a la implementación de dos principios del SUS, promoviendo la atención integral de la salud.

Palabras clave: Terapias complementarias; Enseñando; Enfermería; Sistema único de Salud.

\section{Introdução}

A necessidade de inovação no processo de cuidar em saúde sugere mudanças nos cenários de ensino-aprendizagem. Em 2017, o Conselho Nacional de Saúde (CNS) publicou pressupostos, princípios e diretrizes comuns para a graduação na área da saúde, de forma que é necessária a atualização das Diretrizes Curriculares Nacionais (DCN) para o fortalecimento da formação dos profissionais de saúde e contemplação dos princípios do Sistema Único de Saúde (SUS) da universalidade, equidade e integralidade. Porém, para a implementação dessa premissa no ensino em saúde, existem algumas barreiras pedagógicas (Peixoto et al., 2020).

O ensino em enfermagem, dentro desse cenário, encontra dificuldades no que diz respeito a sua estrutura curricular, marcada pela predominância de um modelo clínico e curativo, com disciplinas fragmentadas e baseadas no modelo flexneriano. Essa forma de ensino se distancia das necessidades da população e da proposta do sistema de saúde público brasileiro, principalmente no que concerne à integralidade em saúde (Mattia et al., 2018).

Nessa perspectiva, é proposto um modelo de cuidar em saúde que possibilita a oferta de assistência não fragmentada: As Práticas Integrativas e Complementares em Saúde (PICS). As PICS consistem em práticas tradicionais que têm o ideal de auxiliar o indivíduo no processo de cura ou prevenção de doenças, a partir da assistência na causa base e na integralidade do ser, mediante cura sintomática e/ou energética (Júnior, 2016). Além de proporcionarem redução sintomática não medicamentosa, essas práticas possuem vários benefícios, dentre eles: alívio da dor e ansiedade, além do aumento do bem-estar (Mendes et al., 2019).

Souza et al. (2020), por exemplo, perceberam que as PICS melhoraram a qualidade de vida de mulheres em sintomas de períodos específicos, tais como a gestação e puerpério. Nelas, houve a diminuição de dores, aumento do bem estar, calma e tranquilidade. Moura e Gonçalves (2020) perceberam que o uso das PICS em pacientes oncológicos provoca a diminuição nos níveis de dor dos pacientes, o que melhora a qualidade de vida dos mesmos.

A partir do reconhecimento das PICS pela Organização Mundial de Saúde (OMS), em 1970, a inclusão, valorização e legitimidade dessas práticas foram incentivadas internacionalmente (Ferreira et al., 2020). No Brasil, são reconhecidas e ofertadas 29 PICS preconizadas politicamente, tais como a Auriculoterapia, Massoterapia, Aromaterapia, entre outras (Dacal, \& Silva, 2018). A institucionalização dessa abordagem se iniciou a partir da criação do SUS e teve sua consolidação com a publicação, em 2006, da Política Nacional de Práticas Integrativas e Complementares (PNPIC). A PNPIC visa estruturar e fortalecer as PICS no SUS através de diretrizes que incentivam sua inserção nos serviços de saúde, assim como o desenvolvimento de estratégias de qualificação profissional e divulgação de informações sobre as práticas (Brasil, 2015).

Vale ressaltar que as PICS possuem um vasto campo de atuação, o que permite seu uso em diferentes níveis de atenção à saúde, tais como na redução da dor no trabalho de parto, da dor oncológica, de sintomas da menopausa, alívio de problemas de cunho mental e afins (Abdoral et al., 2021; Barros et al., 2021; Colomé et al., 2021). Ainda, algumas práticas não tem nenhuma contraindicação, como a aromaterapia vibracional, e outras apresentam poucas contraindicações, a depender do tipo de prática utilizada (Negreiros, 2018).

Apesar dos benefícios reconhecidos, a adesão das mesmas no tratamento em saúde por profissionais e estudantes da enfermagem é reduzido (Almeida et al., 2017). Pesquisas apontam a escassez de debates sobre a temática na maioria das instituições públicas e privadas de ensino em enfermagem (Oliveira et al., 2020). Soma-se à dificuldade de implementação da mesma, tanto em grades curriculares, como em instituições de assistência à saúde, devido ao modelo hospitalocêntrico dominante e ainda vigente (Melo et al., 2013). Dessa forma, é necessário a introdução do ensino das PICS tanto para a graduação em 
enfermagem, como para os profissionais que estão no serviço, de forma a contribuir para a qualidade da assistência e a integralidade do cuidar em saúde (Calado et al., 2019). Destarte, inserir o ensino das PICS contribuirá, ademais, para a consolidação dos princípios que gerem o Sistema Único de Saúde.

Logo, a presente pesquisa tem como objetivo "descrever a experiência do ensino das PICS na graduação em enfermagem", de modo a contribuir com a consolidação científica das mesmas. A relevância da temática proposta consiste na contribuição para a disseminação do conhecimento das medicinas tradicionais, além de incentivar o ensino de uma abordagem em saúde numa perspectiva distinta dos atuais modelos de cuidado, porém com eficácia semelhante. Além disso, a avaliação da satisfação discente frente às temáticas trabalhadas poderá incentivar outras instituições a implementarem o ensino das PICS como atividades regulares.

\section{Método}

Trata-se de um estudo descritivo, do tipo relato de experiência, que aborda as vivências proporcionadas pela disciplina optativa de Práticas Integrativas e Complementares em Saúde. A disciplina foi ofertada entre os meses de março e maio de 2021, no formato remoto, pelo Departamento de Enfermagem de Lagarto/SE, da Universidade Federal de Sergipe- Campus Professor Antonio Garcia Filho.

A disciplina foi ministrada em ambiente virtual, conforme preconiza o plano de Ensino Remoto Emergencial (ERE) da instituição, em decorrência do distanciamento social, necessário durante a pandemia da COVID-19. Os encontros ocorreram de forma síncrona, duas vezes por semana, com duração de três horas cada.

Para a coleta de dados, foi realizada uma observação estruturada das experiências vivenciadas, consulta às anotações pessoais dos autores e debate sobre percepções individuais relativas à disciplina para a prática profissional.

A optativa foi coordenada por três docentes enfermeiras da referida instituição, com experiência na referida temática. Somado a isso, de maneira complementar, foram convidados palestrantes e especialistas de todo Brasil, que atuavam com cada uma das práticas abordadas, para compartilhar suas experiências e conhecimentos sobre as PICS.

Os encontros foram articulados entre apresentações de conteúdo pelas docentes ou palestrantes convidados, junto à apresentação de seminário pelos discentes. Após os momentos síncronos, um formulário feito pelo Google Docs foi passado para ser preenchido com as impressões, dúvidas, comentários e sugestões sobre a aula e o tema. A disciplina seguiu o cronograma programado e abordou as seguintes Práticas Integrativas e sistemas de Medicina Tradicionais, através de palestras em encontros síncronos: Auriculoterapia, Aromaterapia Vibracional, Aromaterapia Tradicional, Constelação Familiar, Metafísica da Saúde, Meditação, Florais de Bach, Radiestesia e Radiônica, Reiki e Barra de Access.

Ademais, foram realizadas apresentações de seminários sobre os temas: Cromoterapia, Reflexologia Podal, Musicoterapia, Ayurveda, Homeopatia, Antroposofia, Fitoterapia e Terapia Comunitária Integrativa. Recursos como slides e vídeos foram utilizados para sistematizar o conteúdo, além da produção de um material criativo e informativo para posterior divulgação, todos embasados por artigos científicos sobre a temática.

\section{Resultados}

Compreende-se as contribuições da oferta da disciplina optativa de PICS para a formação discente em três diferentes esferas: acadêmica, profissional e pessoal.

Referente às contribuições acadêmicas, destacam-se o desenvolvimento da postura e oralidade em público, a habilidade para construção de material educativo e a busca por artigos e referências científicas. Destaca-se, também, o desenvolvimento da curiosidade com as temáticas, devido ao contato com uma carga densa de conteúdo novo, o que despertou a vontade de estudar e buscar, além da necessidade de inovação nas ações em saúde. 
Essas habilidades acadêmicas são essenciais para a formação dos discentes. Através dos seminários, foram trabalhados aspectos da comunicação e da postura ética em apresentações. Tendo em vista que as apresentações são comuns durante a graduação, os seminários contribuíram para o preparo e para a segurança dos discentes ao falar, especialmente para aqueles que ainda estavam no primeiro ano e não tiveram contato com os trabalhos acadêmicos, assim como para aqueles que estavam no último ano e estavam se preparando para apresentar o Trabalho de Conclusão de Curso (TCC).

A construção do material educativo, assim como a busca de referências seguras, contribuiu para o desenvolvimento de atividades acadêmicas, como a escrita e a pesquisa científica. O contato com artigos e estudos proporcionará a ação profissional pautada na Prática Baseada em Evidências (PBE). Ressalta-se, ademais, a possibilidade de compartilhamento com o público externo, o que contribui com a educação em saúde de acadêmicos, profissionais e usuários do SUS e, dessa forma, exercer a integração ensino-serviço-comunidade proposta pelas instituições públicas de ensino.

Quanto ao âmbito profissional, foram trabalhadas as habilidades de trabalho em equipe, da sistematização da assistência e o olhar integral e humanizado ao indivíduo. A referida disciplina contribuiu com o aprendizado de novas formas de agir em saúde, para que se consiga a eficácia, eficiência e efetividade do tratamento dos pacientes, além de causar melhores impactos na assistência do mesmo. Ressalta-se também, o aprendizado em relação ao tratamento da causa base dos problemas em saúde, de forma a ofertar um atendimento com maior nível de integralidade e empatia.

Ressalta-se, ademais, a ciência sobre a regulamentação da atuação da enfermagem nas PICS. A resolução nº 625/2020, por exemplo, aborda as especialidades dos enfermeiros nas PICS, sendo reconhecidas como pós-graduação Lato e Stricto Sensu (Conselho Federal de Enfermagem, 2020) enquanto a n ${ }^{\circ}$ 5858/2018 reconhece a acupuntura como uma especialidade da enfermagem (Conselho Federal de Enfermagem, 2018). Além disso, foi abordado o campo das PICS na execução do processo de enfermagem, através das intervenções e atividades das PICS abordadas na Classificação de Intervenções de Enfermagem (NIC), de modo a reafirmar o uso das PICS pela enfermagem.

Outro benefício observado, dentro do campo acadêmico e profissional, foi a oportunidade de conhecer e tirar dúvidas com profissionais de diversas localizações e que são referências em todo o Brasil. Isso tornou possível experimentar diferentes vivências e pontos de vista sobre os assuntos trabalhados. Além disso, essa conexão com pessoas além da universidade expande a rede de comunicação dos discentes e docentes, o que corrobora com maior divulgação e diálogo sobre as PICS em diferentes estados brasileiros.

No campo pessoal, os discentes foram sensibilizados a realizar uma autoanálise em muitas das atividades explanadas. Ao identificar ferramentas que possam auxiliar no processo de cura e de manutenção da saúde, foi possível perceber como atuar em seu próprio cuidado para que possam então cuidar de outras pessoas. Destaca-se o fortalecimento emocional entre palestrantes, professores e alunos em meio aos variados sentimentos que permeiam a situação pandêmica, tais como o estresse, medo, ansiedade, de forma a impactar positivamente a qualidade de vida de todos. Somado a isso, algumas práticas feitas de modo virtual, como a Meditação e o Reiki, trouxeram benefícios para os indivíduos que assistiram às aulas, proporcionando conforto e clareza mental.

Ocorreu, também, uma satisfação dos discentes com todos os atributos da disciplina, relatada através dos formulários de presença de cada aula e no momento de conclusão, cuja foi de 100\%. Considera-se também o vínculo desenvolvido entre docentes e discente como um ponto positivo dentro dos campos acadêmico, profissional e pessoal, uma vez que expande possibilidades de orientação dentro da universidade. Ainda, essa aproximação foi importante diante do período de medo, ansiedade e tristeza associado à pandemia por COVID-19.

\section{Discussão}

Dada a escassez do ensino das práticas integrativas na graduação em saúde, torna-se relevante a discussão dos achados 
no âmbito acadêmico, profissional e pessoal dos discentes (Melo et al., 2013).

O contato com o inovador dos processos em cuidar em saúde, na perspectiva de desenvolvimento acadêmico, fomenta a curiosidade dos discentes e serve como alicerce para o crescimento de conhecimento nessa área. A curiosidade incentiva a pesquisa científica, bem como promove uma postura acadêmica em consonância às melhores evidências disponíveis, de forma a subsidiar a prática clínica através dos pressupostos da Prática Baseada em Evidências (PBE) (Camargo et al., 2017). A oferta da disciplina traz impactos positivos na formação desses discentes, os capacitando para atuar e divulgar as práticas com embasamento teórico e científico.

Nesse quesito, vale ressaltar ainda que, durante a disciplina, foi trabalhado o pensamento crítico para analisar a aplicação da PBE que, apesar de nortear muitos cuidados em saúde, apresenta limitações quanto às PICS, relacionadas à quantidade de publicações sobre algumas práticas e ao posicionamento da cultura biomédica. É importante haver a sensibilização dentro da universidade para que o uso da PBE aconteça de modo relativizado, uma vez que muitas pesquisas não abordam um cuidado personalizado, o que limita a sua indicação para casos em que se consegue provar não eficácia associada à iatrogenia e dificulta a avaliação de racionalidades médicas vitalistas (Tesser, \& Norman, 2021).

Dessa forma, a apresentação das PICS durante a graduação ocorre como sensibilização para a formação de um cuidado individualizado, que siga princípios bioéticos e de valorização à vida e que busca sempre por novos conhecimentos, mas que não se limita a publicações científicas, visto a incipiência dessas para avaliar contextos e cuidados individualizados.

Dentro das experiências profissionais, destaca-se o aprendizado sobre a importância da oferta do cuidado integral. Essa oferta é essencial para a qualidade assistencial em saúde, de forma a romper com o modelo hospitalocêntrico e fragmentado, além de ressignificar o conceito de saúde pelos próprios usuários. Esse cuidado integral também foi ensinado e executado valorando a espiritualidade, através das práticas de Reiki e de meditação ofertadas na disciplina, além das palestras que enfatizaram tal temática e ofereceram artifícios aos discentes para que o pratiquem profissionalmente no futuro e em seu autocuidado.

O cuidado pautado na espiritualidade é importante para o atendimento de todas as demandas do indivíduo, de forma a se garantir uma maior resolutividade em saúde e coparticipação do indivíduo. Essa abordagem segue os princípios preconizados pela Organização Mundial de Saúde, que afirma que a saúde deve ser analisada na dimensão biológica, psicossocial e espiritual, cuja jamais podem ser vistas de maneira fragmentada. Nessa perspectiva, considerar a espiritualidade e religiosidade do indivíduo ao promover cuidado é uma das formas de buscar a atenção integral, visto que esses fatores influenciam comportamentos e hábitos das pessoas, tendo efeito em seu bem-estar, qualidade de vida e enfrentamento (Cunha, \& Scorsolini-Comin, 2019).

Baseado na importância da abordagem integral, o reconhecimento do Conselho Federal de Enfermagem (COFEN) e de livros importantes para a execução do processo de enfermagem, tais como o NIC, são de extrema importância para a disseminação de novas modalidades de cuidar em saúde, bem como para fomentar o ensino da temática.

Diante disso, as PICS conseguem suprir essa demanda de cuidado integral. As práticas integrativas consideram o indivíduo em sua dimensão global e singular, de forma que não é possível dizer que uma pessoa está 100\% saudável ou doente em seu corpo físico, visto que nesse apenas há a expressão de informações de sua consciência e energia vital. Assim, considerase as patologias como um desequilíbrio de um sistema dinâmico, de forma que os cuidados em saúde devem buscar trazer a harmonia de volta, agindo não apenas no corpo físico (Colomé et al., 2021).

No que concerne às experiências pessoais, ressalta-se o autocuidado, o qual é útil tanto a curto quanto a longo prazo. $\mathrm{O}$ autocuidado é capaz de prevenir problemas típicos em estudantes, tais como estresse patológico em relação às atividades acadêmicas, e a Síndrome de Burnout, típica da enfermagem (Kestenberg et al., 2017; Nogueira et al., 2018). Assim, fornecer estratégias que possibilitam o autocuidado a esse público é prevenir problemas que afetam a qualidade de vida dos mesmos.

Destaca-se ainda, o conhecimento adquirido sobre o campo de ação das PICS, de modo a fomentar o seu uso 
profissionalmente. As PICS servem para diversas finalidades, dentre elas: redução de sintomas oncológicos, ansiedade e/ou dor; auxílio de gestantes, ciclos menstruais e afins. O uso das PICS em pacientes com algias é capaz de melhorar a qualidade de vida, tanto através da diminuição de dor, como na melhora de sintomas mentais, de forma a diminuir a ansiedade e demais sintomas típicos de quem está em processo de tratamento (Moura, \& Gonçalves, 2020; Ferreira et al., 2020).

Ao observar o campo múltiplo de ação, a disciplina consegue ter utilidade para todos os profissionais da saúde, independente da área que escolha trabalhar. Tal premissa, junto à possibilidade de um cuidado mais integral, estimula a adesão das Instituições de Ensino a inserirem como conteúdo obrigatório dos cursos em saúde, tendo em vista que as PICS são ofertadas maiormente como disciplinas optativas. A cultura da oferta dessa disciplina de maneira optativa distancia ou priva o conhecimento de alguns acadêmicos sobre as práticas integrativas. Salienta-se a necessidade dessa inserção, na medida em que um dos desafios para a implementação das PICS citado em pesquisas é justamente a falta de conhecimento (Siedlecki, 2021).

\section{Conclusão}

A disciplina sobre Práticas Integrativas e Complementares em saúde foi exitosa, de modo a cumprir os ideais propostos, dentre eles o auxílio aos discentes na compreensão do que é um cuidado integral em saúde e em como ofertar cuidados pautados na integralidade.

As principais contribuições para a formação discente foram: acadêmica, profissional e pessoal. Os discentes puderam aprender sobre as práticas e visualizar sua aplicabilidade em sua futura prática profissional como enfermeiros, visto que oferta da disciplina feita por docentes enfermeiros facilitou essa aproximação da realidade.

Enfatiza-se, ainda, a importância da expansão desse debate para outras categorias profissionais da área da saúde, já tendo sido ofertada essa disciplina em caráter multidisciplinar na universidade em questão, além da existência de outras optativas abertas a todos os cursos que abordam saúde sob a visão holística.

Conclui-se que o ensino sobre diferentes modalidades de cuidar em saúde é crucial para que ocorra a disseminação das PICS e sua inserção na prática profissional, além de reduzir a principal lacuna referente à insuficiente oferta das práticas nos serviços de saúde: a falta de conhecimento dos profissionais sobre a temática.

\section{Referências}

Abdoral, P. R. G., Freire, A. E. V., Santos, G. F., Rodrigues, L. S., Furtado, F. F., Oliveira, E. M. R., Rezek, P. G., Farias do Couto, M. H. S. H., Brito e Silva, S. C., \& Miranda, C. J. C. P. (2021). Educação em saúde no cuidado de pacientes oncológicos com o uso da auriculoterapia no manejo da dor. Revista Eletrônica Acervo e Saúde, 13(08), 01-9.

Almeida, J. R., Vianini, M. C. S., Silva, D. M., Meneghin, R. A., Souza, G., \& Resende, M. A. (2017). O enfermeiro frente às práticas integrativas e complementares em saúde na estratégia de saúde da família. Revista Eletrônica Acervo e Saúde, 18, 01-7.

Barros, A. L., Pereira, I. P. C., Oliveira, K. R. S. G., Júnior, M. R. S., Real, M. M. F., Pinto, R. J., \& Sousa, S. B. (2021). O uso de Práticas Integrativas e Complementares em Saúde PICS para transtornos mentais. Brazilian Journal of development, 7(08), 78636-46.

Brasil. Ministério da Saúde. (2015). Política Nacional de Práticas Integrativas e Complementares no SUS. Disponível em: Política nacional de práticas integrativas e complementares no SUS: atitude de ampliação de acesso (saude.gov.br).

Calado, R. S. F., Silva, A. A. O. B., Oliveira, D. A. L., Silva, G. A. M., Silva, J. C. B., Silva, L. C., Lemos, M. E. P., \& Santos, R. C. (2019). Ensino das práticas integrativas e complementares na formação em enfermagem. Revista de Enfermagem UFPE online, 13(01), 261-7.

Camargo, F. C., Iwamoto, I. I., Galvão, C. M., Monteiro, D. A. T., Goulart, M. B., \& Garcia, L. A. A. (2017). Modelos para a implementação da prática baseada em evidências na enfermagem hospitalar: revisão narrativa. Texto Contexto e Enfermagem, 24(04), 01-12.

Colomé, I. C. S., Tremea, E., Parcianello, L., Conti, P., Gandin, P. S., Vargas, T. C., Flores, A. M., Frick, E. B., Nogueira, Q. D. S., Lavallós, S. V., \& Garlet, T. M. B. (2021). Auriculoterapia e seus benefícios à saúde: vivências do grupo Pet Saúde Interprofissionalidade. Rede Unida.

Conselho Federal de Enfermagem (COFEN). (2018). Resolução COFEN N o 585/2018. Estabelece e reconhece a acupuntura como especialidade e/ou qualificação do profissional de Enfermagem. Brasília, DF: COFEN.

Conselho Federal de Enfermagem (COFEN). (2020). Resolução 625 de 09 de março de 2020: Altera a resolução Cofen 581 de 11 de julho de 2018 , que 
atualiza, no âmbito do Sistema Cofen/Conselhos Regionais de Enfermagem, os procedimentos para Registro de Títulos de Pós-Graduação Lato e Stricto Sensu concedido a Enfermeiros e aprova lista de especialidades. Brasília, DF: COFEN.

Cunha, V. F., Scorsolini-Comin, F. (2017). A dimensão religiosidade/espiritualidade na Prática Clínica: revisão Integrativa da literatura científica. Psicologia: Teoria e Pesquisa, 35, 01-12.

Dacal, M. P. O., \& Silva, I. S. (2018). Impactos das práticas integrativas e complementares na saúde de pacientes crônicos. Saúde Debate, 42(118), 724-735.

Ferreira, S. K. S., Cunha, I. P., Meneghin, M. C., \& Checci, M. H. R. (2020). Política nacional de práticas integrativas e complementares no Sistema Único de Saúde. Revista Faipe, 10(01), 21-39.

Júnior, E. T. (2016). Práticas Integrativas e complementares em saúde, uma nova eficácia para o SUS. Estudos Avançados, 30(86), 99-112.

Kestenberg, C. C. F., Rosa, B. M. S., Silva, A. V., Fabri, J. M. G., \& Regazi, I. C. R. (2017). Estresse em graduandos de enfermagem. Revista de Enfermagem UFRJ, 25, 01-6.

Mattia, B. J., Kleba, M. E., Prado, M. L. (2018). Formação em enfermagem e a prática profissional: uma revisão integrativa da literatura. Revista Brasileira de Enfermagem, 71(4), 2039-2049.

Melo, S. C. C., Santana, R. G., Santos, D. C., \& Alvim N. A. T. (2013). Práticas complementares de saúde e os desafios de sua aplicabilidade no hospital: visão de enfermeiros. Revista Brasileira de Enfermagem, 66(06), 840-6.

Mendes, D. S., Moraes, F. S., Lima, G. O., Silva, P. R., Cunha, T. A., Crosseti, M. G. O., \& Riegel F. (2019). Benefícios das práticas integrativas e complementares no cuidado de enfermagem. Journal Helps NPEPS, 4(01), 302-18.

Moura, A. C. A., \& Gonçalves, C. C. S. (2020). Práticas integrativas e complementares para alívio ou controle da dor em oncologia. Revista de Enfermagem Contemporânea, 9(01), 101-08.

Negreiros, M. V. (2018). Uma antropologia de incertezas entre a biomedicina e a eficácia das práticas de yoga e meditação no SUS. Dissertação de mestrado. Universidade Federal de São Paulo. Guarulhos, SP, Brasil.

Nogueira, L. S., Sousa, R. G. C., Guedes, E. S., Santos, M. A., Turrini, R. N. T., \& Cruz, D. A. L. N. (2018). Burnout e ambiente de trabalho de enfermeiros em instituições públicas de saúde. Revista Brasileira de Enfermagem, 71(02), 358-65.

Oliveira, T. A., Assis, T. A. A., Macedo, J. A., Silva, I. A., \& Almeida, E. A. (2020). Ensino das Práticas Integrativas e Complementares nos cursos de enfermagem do Estado de São Paulo. Revista Nursing, 23(266), 4392-4396.

Peixoto, M. T., Miranda, A. C., \& Peixoto, M. T. (2020). Formação para a Atenção Primária à Saúde. JMPHC| Journal of Management \& Primary Health Care, $12,1-10$.

Siedlecki, S. (2021). Terapias Complementares e alternativas em programas acadêmicos de enfermagem: é necessário mais educação? Complementary Therapies in Clinical Pratice, 43, 101327.

Souza, V. S., Machado, G. N., Arrué, A. M., Luzardo, A. R., Jantsch, L. B., \& Danski, M. T. R. (2020). As Práticas Integrativas e Complementares na atenção à saúde da mulher. Research, Society em development, 9(08), 01-13.

Tesser, C. D., \& Norman, A. H. (2021). Prevenção quaternária e práticas integrativas e complementares (II): aproximação contextual. Revista Brasileira de Medicina de Família e Comunidade, 16(43), 2566-66. 